\title{
Expanded Noninvasive Prenatal Testing for Chromosomal aneuploidies and Copy Number Variants in a Cohort of 16128 Single Pregnancies
}

Jing He ( $\square$ hj0113@yeah.net )

Gansu Province Maternal and Child Health Care Hospital https://orcid.org/0000-0002-9881-1025

Xuan Feng

Gansu Province Maternal and Chid Health Care Hospital

Xing Wang

Gansu Province Maternal and Child Health Care Hospital

Qinghua Zhang

Gansu Province Maternal and Child Health Care Hospital

Lei Zheng

Gansu Province Maternal and Chid Health Care Hospital

Pengwu Lin

Gansu Province Maternal and Child Health Care Hospital

Shengju Hao

Gansu Province Maternal and Child Health Care Hospital

\section{Research Article}

Keywords: noninvasive prenatal testing, copy number variants, Sex chromosome abnormalities, maternal copy number variants, Advanced maternal age.

Posted Date: August 20th, 2021

DOI: https://doi.org/10.21203/rs.3.rs-810119/v1

License: @ (1) This work is licensed under a Creative Commons Attribution 4.0 International License. Read Full License 


\section{Abstract}

Background: Noninvasive prenatal testing (NIPT) is based on second-generation genomic sequencing technology to scan cell-free fetal DNA originating from the placenta in maternal plasma. As the depth of sequencing increases, it can be used to focus on chromosomal aneuploidies, copy number variants (CNVs), and monogenic diseases. It can significantly improve the accuracy of prenatal screening and reduces the number of invasive testing.

Methods: In this study, we retrospectively analyzed 16128 naturally conceived singleton pregnancies who underwent expanded NIPT to calculate the true positive rate (TPR) of chromosomal aneuploidies and CNVs, and analyzed the potential influence of maternal sex chromosome abnormalities (SCAs) and maternal CNVs on expanded NIPT results.

Results: After invasive prenatal diagnosis and follow-up, 103 pregnancies were found to be true-positive, including 73 cases of chromosomal abnormalities and 30 cases of CNVs. The TPR of T21 was $84.62 \%$, T18 was $50.00 \%$, T13 was $22.22 \%$, SCA was $34.06 \%$, and CNVs was $40.28 \%$. In addition, we found that the positive rate of aneuploidies increased with maternal age and that maternal SCAs accounted for $13.33 \%$ of the 60 false positive cases of SCAs.

Conclusion: Expanded NIPT showed high sensitivity and specificity in detecting diseases of chromosomal abnormalities. It also shows good performance in detecting CNVs, but maternal SCAs and CNVs confused some NIPT results, indicating it is still necessary to study the potential maternal influence on expanded NIPT results and to report related clinical validation studies.

\section{Background}

The clinical use of noninvasive prenatal testing (NIPT) using maternal plasma to detect fetal genetic material was made possible by the discovery of cell-free fetal DNA (cffDNA) in the maternal circulation in 1997(1)and the development of next-generation sequencing in 2008(2). This technological innovation significantly reduces the number of invasive tests, and increases the efficiency of invasive prenatal screening(3). A large number of clinical studies have shown that NIPT has a high sensitivity and specificity for diseases of chromosomal aneuploidy. The true-positive rates range of T21 (Down's syndrome) was 65-95\%, T18 (Edward's syndrome) was 47-85\%, and T13 (Patau syndrome) was $12-62 \%(4-6)$. As the depth of sequencing increases and the calculation methods change, the focus is on aneuploidies, copy number variants (CNVs), and monogenic diseases. CNVs cause microdeletion/microduplication syndromes (MMS), which are unlikely to be detected by ultrasound examination and have a much higher incidence than Down syndrome(7), accounting for $1-2 \%$ of newborn congenital abnormalities(8). Studies have suggested that expanded NIPT yielded high positive predictive values (PPV) for common aneuploidies and DiGeorge syndrome, and moderate PPVs for other MMS(9), However, the rate of false positive and false negative results makes the implementation of the expanded NIPT more challenging, therefore requiring validation in clinical practice. In this retrospective study, we analyzed 16128 patients with naturally conceived singleton pregnancies using expanded NIPT and analyzed the performance of expanded NIPT as a screening test for fetal aneuploidies and CNVs. We also calculated the influence of maternal age, Sex chromosome abnormalities (SCAs), and maternal copy number variants (MCNV) on the positive rate of fetal aneuploidies and CNVs.

\section{Results}

\subsection{Pregnancy characteristics}

A total of 16128 naturally conceived singleton pregnancies were included in this study. The maternal age ranged from 15 to 55 years-old and the pregnancy gestations ranged from $11^{+0}$ to $32^{+6}$ weeks. Of all the participants in the study, 2735 had a history of more than two spontaneous abortions or pregnancy have been pregnant or birth defect, that called had a history of adverse pregnancy and childbirth (16.96\%). Among the 16128 participants who underwent expanded NIPT, 1201 (7.45\%) showed fetal structural abnormalities by Bultrasound(including NT $\geq 3 \mathrm{~mm}), 1785$ (11.07\%) showed a high risk of serological screening, $5143(31.89 \%)$ showed a critical risk of serological screening, 4889 (30.31\%) had advanced maternal age (age $\geq 35)$, 2295(14.23\%) showed no serology screening, and 815 (5.05\%) had no clinical indications. (Table 1). 
Maternal characteristics and gestational age.

\begin{tabular}{|c|c|c|}
\hline Maternal age at NIPT (years) & No./N = 16128 & Rate (100\%) \\
\hline$<30$ & 6062 & 37.59 \\
\hline $30-34$ & 4916 & 30.48 \\
\hline $35-40$ & 4858 & 30.12 \\
\hline$\geq 41$ & 292 & 1.81 \\
\hline Range & $15-55$ & \\
\hline Gestational age at NIPT (weeks) & No. & Rate $(100 \%)$ \\
\hline $11-15^{+6}$ & 4612 & 28.6 \\
\hline $16-22^{+6}$ & 9782 & 60.65 \\
\hline $23-32^{+6}$ & 1734 & 10.75 \\
\hline Range & $11-32^{+6}$ & \\
\hline History of adverse pregnancy and childbirth & No./N = 16128 & Rate (100\%) \\
\hline Yes & 2735 & 16.96 \\
\hline No & 13393 & 83.04 \\
\hline Clinical features & No./N = 16128 & Rate $(100 \%)$ \\
\hline Fetal structural abnormalities by B-ultrasound & 1201 & 7.45 \\
\hline High risk of serological screening & 1785 & 11.07 \\
\hline Critical risk of serological screening & 5143 & 31.89 \\
\hline Advanced maternal age ( $\geq 35$ years) & 4889 & 30.31 \\
\hline No serology screening & 2295 & 14.23 \\
\hline No clinical indications* & 815 & 5.05 \\
\hline
\end{tabular}

\subsection{The performance of expanded NIPT}

Of the 16128 participants that underwent expanded NIPT, 287 abnormal results were detected, and diagnostic testing by karyotyping and CNV-Seq was used to verify the abnormal results. Among the 287 cases, 60 refused prenatal diagnosis and the remaining 227 cases were verified and followed up with the following results: 103 true positives (33 cases of T21, 7 of T18, 2 of T13, 31 of SCAs, 30 of CNVs); 124 false positives ( 6 cases of T21, 7 of T18, 7 of T13, 60 of SCA, 44 of CNVs); and one false negative (T21). Moreover, the true positive rate (TPR) and the false positive rate (FPR)for each test was assessed. For trisomy 21 (T21), the TPR was 84.62\% (95\%Cl, 73.30\%-95.94\%), the FPR was $15.38 \%$, For trisomy 18 (T18), the TPR was $50.00 \%$ (95\%Cl, 23.81\%-76.19\%), the FPR was $50.00 \%$. For trisomy 13 (T13), the TPR was $22.22 \%(95 \% \mathrm{Cl}, 4.94 \%-49.38 \%)$, the FPR was $77.78 \%$. For SCAs, the TPR was $34.06 \%(95 \% \mathrm{Cl}, 24.32 \%-43.79 \%)$, the FPR was $65.94 \%$. For CNVs, the TPR was $36.25 \%(95 \% \mathrm{Cl}, 25.30 \%-47.20 \%)$, the FPR was $63.75 \%$. (Table 2). 
Table 2

Performance of expanded NIPT.

\begin{tabular}{|llllll|}
\hline NIPT & T21 & T18 & T13 & SCAs & CNVs \\
\hline Positive & 46 & 18 & 10 & 121 & 98 \\
\hline Unverified & 7 & 4 & 1 & 30 & 18 \\
\hline TP & 33 & 7 & 2 & 31 & 29 \\
\hline FP & 6 & 7 & 7 & 60 & 51 \\
\hline TPR & 84.62 & 50 & 22.22 & 34.06 & 36.25 \\
\hline FPR & 15.38 & 50 & 77.78 & 65.94 & 63.75 \\
\hline
\end{tabular}

\subsection{TPRs of chromosomal aneuploidies according to pregnancy characteristics}

As shown in Table 3, different pregnancy characteristics correspond to different TPRs. The total TPR of T21 was $84.62 \%$ (95\%Cl, 73.30\%-95.94\%). In both the high risk of serological screening group and the no serology screening group, the TPRs of T21 were the highest at $100 \%$, while the TPRs of T21 in the advanced maternal age group, the B-Ultrasound indicated abnormalities group, and the Critical risk of serological screening group were $92.86 \%, 83.33 \%$ and $66.67 \%$, respectively. The total TPR of SCAs was $34.06 \%$ (95\%Cl, $27.06 \%-41.06 \%$ ), with the highest being $50.00 \%$ in the Ultrasound indicated abnormalities. The TPRs of T18 in the high risk of serological screening group, the critical risk of serological screening group, advanced maternal age group, and the no serology screening group were $16.67 \%, 30.77 \%, 39.28 \%$, and $28.57 \%$, respectively. Among the 153 positive cases which underwent invasive prenatal diagnosis, the number of true positive cases was 73 , the number of false positive case was 80 , and the overall TPR of aneuploidies was $47.71 \%$. The TPRs of aneuploidies in the B-Ultrasound indicated abnormalities group, the high risk of serological screening group, the Critical risk of serological screening group, the advanced maternal age group, and the no serology screening group were $55.56 \%, 36.84 \%, 40.90 \%, 58.00 \%$, and $35.00 \%$, respectively.

Table 3

TPRs of chromosomal aneuploidies according to pregnancy characteristics.

\begin{tabular}{|c|c|c|c|c|c|c|c|c|c|c|c|c|c|c|c|}
\hline \multirow{2}{*}{$\begin{array}{l}\text { Clinical } \\
\text { features }\end{array}$} & \multicolumn{3}{|c|}{ T21 } & \multicolumn{3}{|c|}{ T18 } & \multicolumn{3}{|l|}{ T13 } & \multicolumn{3}{|c|}{ SCAs } & \multicolumn{3}{|c|}{ Total } \\
\hline & TP & FP & $\begin{array}{l}\text { TPR } \\
(\%)\end{array}$ & TP & FP & $\begin{array}{l}\text { TPR } \\
(\%)\end{array}$ & $\mathrm{TP}$ & FP & $\begin{array}{l}\text { TPR } \\
(\%)\end{array}$ & TP & FP & $\begin{array}{l}\text { TPR } \\
(\%)\end{array}$ & TP & FP & $\begin{array}{l}\text { TRP } \\
(\%)\end{array}$ \\
\hline $\begin{array}{l}\text { Ultrasound } \\
\text { indicated } \\
\text { abnormalities }\end{array}$ & 5 & 1 & 83.33 & & & & & 2 & & 5 & 5 & 50.00 & 10 & 8 & 55.56 \\
\hline $\begin{array}{l}\text { High risk of } \\
\text { serological } \\
\text { screening }\end{array}$ & 4 & & 100 & & 1 & & 1 & 1 & 50.00 & 2 & 10 & 16.67 & 7 & 12 & 36.84 \\
\hline $\begin{array}{l}\text { Critical risk of } \\
\text { serological } \\
\text { screening }\end{array}$ & 8 & 4 & 66.67 & 1 & 2 & 33.33 & 1 & 2 & 33.33 & 8 & 18 & 30.77 & 18 & 26 & 40.90 \\
\hline $\begin{array}{l}\text { Advanced } \\
\text { maternal age } \\
(\geq 35)\end{array}$ & 13 & 1 & 92.86 & 5 & 2 & 71.43 & & 1 & & 11 & 17 & 39.28 & 29 & 21 & 58.00 \\
\hline $\begin{array}{l}\text { No serology } \\
\text { screening }\end{array}$ & 3 & & 100 & & 2 & & & 1 & & 4 & 10 & 28.57 & 7 & 13 & 35.00 \\
\hline $\begin{array}{l}\text { No clinical } \\
\text { indications }\end{array}$ & I & I & & 1 & / & & I & / & & 1 & I & & 2 & I & \\
\hline Total & 33 & 6 & 84.62 & 7 & 7 & 50.00 & 2 & 7 & 22.22 & 31 & 60 & 34.06 & 73 & 80 & 47.71 \\
\hline
\end{tabular}

\subsection{The influence of maternal age on the positive rate of fetal aneuploidies and CNVs}

As shown in Fig. 1, we divided the study participants by maternal age into four groups to analyze the influence of maternal age on the positive rate of fetal aneuploidies and CNVs. The following positive rates were determined for the different maternal age groups: the < 30 years group was $0.36 \%(95 \% \mathrm{Cl}, 0.21 \%-0.51 \%)$, the positive rate of the $30-34$ years group was $0.47 \%(95 \% \mathrm{Cl}, 0.29 \%-0.66 \%)$, the $35-40$ years group was $0.66 \%(95 \% \mathrm{Cl}, 0.43 \%-0.89 \%)$, the $\geq 41$ years group was $2.05 \%(95 \% \mathrm{Cl}, 0.42 \%-3.68 \%)$. A Chi-square test was used to analyze the 
significance of the differences between the different groups. For chromosome aneuploidies, the positive rate of the advance maternal age group (the 35-40 group and the $\geq 41$ group) was higher than the $<35$ group ( $<30$ group and $30-34$ group), and the difference was statistically significant $(X 2=8.651, p=0.003<0.05)$. For $C N V$ s, the difference between the advance maternal age group $(35-40$ group and $\geq$ 41 group) and the $<35$ years group ( $<30$ group and $30-34$ group) was not statistically significant $(x 2=0.000, p=1.000>0.05)$. The total positive rate increased with maternal age, and the positive rate of the advance maternal age group (35-40 group and $\geq 41$ group) was higher than the $<35$ group ( $<30$ group and $30-34$ group), and the difference was statistically significant $(\chi 2=4.409, p=0.036<0.05)$.

\subsection{The potential influence of parental CNVs on fetal CNVs}

Besides T21 T18 and T13, we analyzed other chromosomal aneuploidies and CNVs among the 16128 samples. A total of $98(0.61 \%)$ cases were detected to have abnormal CNVs results, 80 cases underwent invasive prenatal diagnosis, while 18 patients refused amniotic fluid puncture. Of the 80 cases that underwent invasive prenatal diagnosis, 29(36.25\%) of them were true positives (Table 4), 51(63.75\%) cases were false positives (including nine false positive cases where abnormal results were detected in the mother's peripheral blood, while the fetal amniotic fluid was normal). Among the 29 cases where abnormal results were detected in the fetal amniotic fluid, 27 cases underwent Parental peripheral blood verification. Additionally, among the 27 cases, $13(48.15 \%)$ cases occurred because of genetic mutations, while $14(51.85 \%)$ cases were inherited from parents. Among the 14 cases, 13 cases were inherited from the mother and 1 case was inherited from the father. Among the 29 cases where abnormal results were detected in the fetal amniotic fluid, $9(31.03 \%)$ cases were identified as

syndrome diseases or pathogenicity, and $15(51.727 \%)$ cases had unknown pathogenicity, and $5(17.24 \%)$ cases were benign, according to the ACMG guidelines. 
Table 4

The true positive of CNVs.

\begin{tabular}{|c|c|c|c|c|c|}
\hline \multirow[t]{2}{*}{ Case } & \multirow[t]{2}{*}{ MG } & \multirow[t]{2}{*}{ NIPT results } & \multicolumn{2}{|l|}{ prenatal diagnosis } & \multirow[t]{2}{*}{ Parents' CNV-seq } \\
\hline & & & CNV-seq & karyotype & \\
\hline \multicolumn{6}{|c|}{ A. Genetic mutations } \\
\hline 1 & 32 & $\begin{array}{l}\text { 7p22.3-p11.2 dup } \\
\text { 55.6Mb,7q11.21-q36.3 dup } \\
\text { 92.3Mb }\end{array}$ & $47, \mathrm{XN}+7[10 \%] / 46, \mathrm{XN}[90 \%]$ & $46, \mathrm{XN}$ & normal \\
\hline \multirow[t]{2}{*}{2} & \multirow[t]{2}{*}{27} & \multirow{2}{*}{$\begin{array}{l}\text { 7p22.3-p11.2 dup } \\
\text { 55.6Mb,7q11.21-q36.3 dup } \\
\text { 92.3Mb }\end{array}$} & \multirow[t]{2}{*}{$47, \mathrm{XN}+7(40 \%) / 46, \mathrm{XN}(60 \%)$} & $47, \mathrm{XN}+7[40 \%]$ & \multirow[t]{2}{*}{ normal } \\
\hline & & & & $/ 46, \mathrm{XN}[60 \%]$ & \\
\hline 3 & 42 & $\begin{array}{l}\text { 7q36.2-q36.3 dup4.3Mb, 14q11.2- } \\
\text { q21.3 dup 27.4Mb }\end{array}$ & $\begin{array}{l}\text { 7q36.2-q36.3 dup4.44Mb, 14q11.2- } \\
\text { q21.3 dup } 29.06 \mathrm{Mb}\end{array}$ & $\begin{array}{l}46, \mathrm{XN}, \operatorname{dup}(14) \\
\text { (q11.2-q21.3) }\end{array}$ & normal \\
\hline 4 & 25 & 10q.24.2-q26.3 dup 35.8Mb & 10q24.1-q26.3 dup 37.2Mb & $\begin{array}{l}46, X N, \operatorname{dup}(10) \\
\text { (q24.1-q26.3) }\end{array}$ & normal \\
\hline 5 & 27 & 12p13.33-p11.1 dup 34.3Mb & 12p13.33-p11.1 dup34.7Mb & $\begin{array}{l}\text { 46, XN, dup (12) } \\
\text { (p13.3-p11.1) }\end{array}$ & normal \\
\hline 6 & 34 & 13q33.3-q34del4.2mb, & 13q33.3-q34del4.86Mb, & $46, \mathrm{XN}$ & normal \\
\hline 7 & 27 & 18p11.32-p11.21 del 13.5 & 18p11.32-p11.21del 14.86Mb & $46, \mathrm{XN}$ & normal \\
\hline 8 & 38 & 22q11 deletion syndrome & $22 q 11.21$ del $2.58 \mathrm{Mb}$ & $46, \mathrm{XN}$ & normal \\
\hline 9 & 30 & 6p24.3-p22.3 del 5.2Mb & $6 \mathrm{p} 24.2-\mathrm{p} 22.3$ del $5.12 \mathrm{Mb}$ & $46, \mathrm{XN}$ & normal \\
\hline 10 & 28 & 6q23.3-q24.1 dup 2.0Mb & 6q23.3-q24.1 dup 2.88Mb & $46, \mathrm{XN}$ & normal \\
\hline 11 & 29 & 10q22.3-q23.1 del 4.5Mb & $10 q 22.3-q 23.1 \mathrm{del} 4.46 \mathrm{Mb}$ & $46, \mathrm{XN}$ & normal \\
\hline 12 & 24 & 4q35.2 dup 2.0Mb & 4q35.2 dup 1.92Mb & $\begin{array}{l}45, \mathrm{XN}, \text { rob }(13 ; 14) \\
(\mathrm{q} 10 ; \mathrm{q} 10)\end{array}$ & normal \\
\hline 13 & 36 & 8p23.1 dup 3.8Mb & 8p23.1 dup 3.76Mb & $46, \mathrm{XN}$ & normal \\
\hline \multicolumn{6}{|c|}{ B. Inherited from parents } \\
\hline 14 & 27 & $22 q 11.21$ dup $2.4 \mathrm{Mb}$ & $22 q 11$ dup $2.5 \mathrm{Mb}$ & $46, \mathrm{XN}$ & $\begin{array}{l}\text { 22q11 duplication } \\
\text { syndrome (M) }\end{array}$ \\
\hline 15 & 35 & 16p13.11-p12.3 dup 2.7Mb & 16p13.11-p12.3 dup 2.64Mb & $46, \mathrm{XN}$ & $\begin{array}{l}\text { 16p13.11-p12.3 dup } \\
2.64 M b(M)\end{array}$ \\
\hline 16 & 32 & $16 q 21 \mathrm{del} 4.0 \mathrm{Mb}$ & $16 q 21$ del3.98Mb & $46, \mathrm{XN}$ & $\begin{array}{l}16 q 21 \text { del 3.98Mb } \\
(M)\end{array}$ \\
\hline 17 & 24 & 1p36.33-p36.32 dup 2.4Mb & 1p36.33-36.32 dup 2.26Mb & $46, \mathrm{XN}$ & $\begin{array}{l}\text { 1p36.33-36.32 dup } \\
2.38 \mathrm{Mb}(\mathrm{M})\end{array}$ \\
\hline 18 & 32 & $2 \mathrm{p} 22.3$ dup $2.3 \mathrm{Mb}$ & $2 \mathrm{p} 22.3$ dup $2.20 \mathrm{Mb}$ & $46, \mathrm{XN}$ & $\begin{array}{l}2 \mathrm{p} 22.3 \text { dup } 2.20 \mathrm{Mb} \\
(\mathrm{M})\end{array}$ \\
\hline 19 & 32 & 3q11.1-q11.2 dup3.2Mb & $3 q 11.1-q 11.2$ dup 3.06Mb & $46, X N$, inv (9) & $\begin{array}{l}3 q 11.1-q 11.2 \text { dup } \\
3.10 \mathrm{Mb}(\mathrm{M})\end{array}$ \\
\hline 20 & 28 & $4 q 34.3$ dup $2.0 \mathrm{Mb}$ & 4q34.3 dup $1.66 \mathrm{Mb}$ & $46, \mathrm{XN}$ & $\begin{array}{l}\text { 4q34.3 dup 1.62Mb } \\
\text { (M) }\end{array}$ \\
\hline 21 & 26 & $4 q 34.3$ dup $2.2 \mathrm{Mb}$ & 4q34.3 dup 1.62Mb & $46, \mathrm{XN}$ & $\begin{array}{l}\text { 4q34.3 dup } 1.72 \mathrm{Mb} \\
(\mathrm{M})\end{array}$ \\
\hline 22 & 26 & $4 q 34.3$ dup $2.4 \mathrm{Mb}$ & $4 q 34.3$ dup $2.28 \mathrm{Mb}$ & $46, \mathrm{XN}$ & $\begin{array}{l}\text { 4q34.3 dup } 2.22 \mathrm{Mb} \\
(\mathrm{M})\end{array}$ \\
\hline 23 & 37 & $7 p 21.3$ dup $3.1 \mathrm{Mb}$ & $7 p 21.3$ dup $0.7 \mathrm{Mb}$ & $46, \mathrm{XN}$ & $\begin{array}{l}\text { 7p21.3 dup } 1.4 \mathrm{Mb} \\
(\mathrm{M})\end{array}$ \\
\hline
\end{tabular}




\begin{tabular}{|c|c|c|c|c|c|}
\hline \multirow[t]{2}{*}{ Case } & \multirow[t]{2}{*}{ MG } & \multirow[t]{2}{*}{ NIPT results } & \multicolumn{2}{|l|}{ prenatal diagnosis } & \multirow[t]{2}{*}{ Parents' CNV-seq } \\
\hline & & & CNV-seq & karyotype & \\
\hline 24 & 22 & 8p23.2 dup 2.3Mb & $8 p 23.2$ dup $2.22 \mathrm{Mb}$ & $46, X N$ & $\begin{array}{l}8 \mathrm{p} 23.2 \text { dup } 2.22 \mathrm{Mb} \\
\text { (M) }\end{array}$ \\
\hline 25 & 33 & 8p23.2 dup 2.6Mb & $8 p 23.2$ dup $2.26 \mathrm{Mb}$ & $46, X N$ & $\begin{array}{l}8 \mathrm{p} 23.2 \text { dup } 2.26 \mathrm{Mb} \\
(\mathrm{M})\end{array}$ \\
\hline 26 & 34 & $8 q 24.21-q 24.22$ dup 2.0Mb & 8q24.21-q24.22 dup 1.3Mb & $46, X N$ & $\begin{array}{l}8 q 24.21-q 24.22 \text { dup } \\
1.3 \mathrm{Mb}(\mathrm{M})\end{array}$ \\
\hline 27 & 23 & $10 \mathrm{q} 21.1 \mathrm{del} 3.1 \mathrm{Mb}$ & $10 q 21.1$ del3.04Mb & $46, X N$ & $\begin{array}{l}10 \mathrm{q} 21.1 \text { del3.04Mb } \\
(\mathrm{F})\end{array}$ \\
\hline \multicolumn{6}{|c|}{ C. Parents' CNVs Unverified } \\
\hline 28 & 24 & $3 q 11.2$ dup $2.6 \mathrm{Mb}$ & $3 q 11.1-q 11.2$ dup $3.16 \mathrm{Mb}$ & & / \\
\hline 29 & 25 & 3p12.3-p12.2 dup 2.5Mb & 3p12.3-p12.2 dup 2.5Mb & & / \\
\hline
\end{tabular}

\subsection{Potential influence of maternal SCAs and CNVs on expanded NIPT}

Among the results of expanded NIPT, there were eight cases where we detected normal results in the fetal amniotic fluid, but the FISH analysis showed SCAs in the maternal peripheral blood (Table 5.A). Of the 60 false positive cases of fetal SCAs, maternal SCAs accounted for $13.33 \%$. Among expanded NIPT, there were 9 cases where we detected normal results in the fetal amniotic fluid, but the CNV-seq showed abnormal results in the maternal peripheral blood (Table 5.B).

Table 5

The influence of maternal SCAs and CNVs on expanded NIPT results

\begin{tabular}{|c|c|c|c|}
\hline Case & NIPT results & prenatal diagnosis & Maternal peripheral blood \\
\hline \multicolumn{4}{|c|}{ A. Maternal Potential influence of SCAs } \\
\hline 1 & ChrX- & $46, \mathrm{XN}$ & $46, X X[68 \%] / 47, X X X[32 \%]$ \\
\hline 2 & ChrX+ & $46, \mathrm{XN}$ & $45, X 0[26 \%] / 46, X X[74 \%]$ \\
\hline 3 & ChrX- & $46, \mathrm{XN}$ & $45, \mathrm{X0}[13 \%] / 46, \mathrm{XX}[87 \%]$ \\
\hline 4 & $\operatorname{ChrX+(Y)}$ & $46, \mathrm{XN}$ & 47, XXX \\
\hline 5 & ChrX- & $46, \mathrm{XN}$ & $45, \mathrm{X0}[10 \%] / 46, \mathrm{XX}[90 \%]$ \\
\hline 6 & $\operatorname{ChrX+(Y)}$ & $46, \mathrm{XN}$ & $46, X X[33 \%] / 47, X X X[67 \%]$ \\
\hline 7 & ChrX- & $46, \mathrm{XN}$ & $45, X 0[30 \%] / 46, X X[70 \%]$ \\
\hline 8 & ChrX- & $46, \mathrm{XN}$ & $45, \mathrm{X0}[20 \%] / 46, \mathrm{XX}[80 \%]$ \\
\hline \multicolumn{4}{|c|}{ B. Maternal Potential influence of copy number variations } \\
\hline 1 & 16p13.11-p12.3 dup 2.7Mb & $46, \mathrm{XN}$ & 16p13.11-p12.3 dup 2.5Mb \\
\hline 2 & 1q21.1-q21.2 dup 2.0Mb & $46, \mathrm{XN}$ & 1q21.1 recurrent micro-duplication \\
\hline 3 & $1 \mathrm{q} 43$ dup $2.2 \mathrm{Mb}$ & $46, X N$ & $1 \mathrm{q} 43$ dup $0.34 \mathrm{Mb}$ \\
\hline 4 & $2 \mathrm{p} 12-\mathrm{p} 11.2$ dup $2.1 \mathrm{Mb}$ & $46, \mathrm{XN}$ & $2 \mathrm{p} 12$ dup $1.1 \mathrm{Mb}$ \\
\hline 5 & 4p13-p12 del 2.5Mb & $46, \mathrm{XN}$ & 4p13-p12 del 2.34Mb \\
\hline 6 & $5 q 34$ del $3.6 \mathrm{Mb}$ & $46, \mathrm{XN}$ & $5 q 34$ del $3.5 \mathrm{Mb}$ \\
\hline 7 & 6p21.33-p21.32 dup 2.6Mb & $46, \mathrm{XN}$ & $6 \mathrm{p} 21.32$ dup $0.26 \mathrm{Mb}$ \\
\hline 8 & 8p23.2 dup 2.3Mb & $46, \mathrm{XN}$ & 8p23.2 dup $2.26 \mathrm{Mb}$ \\
\hline 9 & 8q21.13 dup $2.1 \mathrm{Mb}$ & $46, \mathrm{XN}$ & 8q21.13 dup 1.9Mb \\
\hline
\end{tabular}




\section{Discussion}

Compared to traditional prenatal screening methods based on serological screening and ultrasound screening to assess fetal chromosomal abnormalities, NIPT is a more accurate prenatal screening tool. The detection rate of chromosomal abnormalities of traditional prenatal screening is $50 \%-95 \%(10)$, while the sensitivity and specificity of NIPT for fetal trisomy's 21,18 , and 13 are higher than $99 \%(11)$. In our study, in addition to invasive prenatal diagnosis, we also conducted follow-up. Besides the unverified cases, we found 20 false positive cases and one false negative case of T21. The false positive rate and the specificity of expanded NIPT for fetal trisomy's 21,18 , and 13 was found to be $0.12 \%$ and $99.88 \%$, respectively. The false negative rate and the sensitivity of expanded NIPT for fetal trisomy's 21,18 , and 13 was found to be $0.0062 \%$ and $99.99 \%$, respectively. Our results also show that the TPR of T21 was $84.62 \%(95 \% \mathrm{Cl}, 73.30 \%-95.94 \%)$, the TPR of T18 was $50.00 \%(95 \% \mathrm{Cl}, 23.81 \%-76.19 \%)$, the TPR of T13 was $22.22 \%(95 \% \mathrm{Cl}, 4.94 \%-49.38 \%)$, and the TPR of CNVs was $40.54 \%(95 \% \mathrm{Cl}$, 29.35\%-51.73\%). Expanded NIPT is not only more accurate but also avoids unnecessary invasive prenatal diagnosis methods which may result in approximately $0.1-0.3 \%$ procedure-related pregnancy loss(12), and more and more pregnant women are willing to choose expanded NIPT(13). With the deepening of sequencing, the expanded NIPT, which detects aneuploidies and genome-wide MMS caused by CNVs, has become available. Studies have shown that about $80 \%$ of pregnant couples in the Netherlands are willing to choose whole genome testing instead of common trisomies(14).

According to China's guidelines for NIPT published on October 27, 2016, NIPT should be used with caution for pregnant women older than 35 and for patients with a high risk of serological screening. However, our findings reveal that many of them opted for NIPT to avoid invasive prenatal diagnosis. This observation is also supported by the study by Tian et al(15). Among 16128 pregnant women, $4889(30.31 \%)$ of them were older than 35 , and 1785 (11.07\%) of them showed a high risk of serological screening. Simultaneously, in the older than 35 group, the TPR of T21 was $92.86 \%$, the TPR of T18 was $71.43 \%$, and the TPR of SCAs was $39.28 \%$. As such, the expanded NIPT reduced the incidence of invasive procedures. Consistent with previous studies(16), we found that the prevalence of fetal aneuploidies increased with the maternal age. Our study shows that for chromosomal aneuploidies, the incidence of abnormal results tends to increase with the maternal age. Taking 35 as the node, the positive rate of abnormality in advanced maternal age group is higher than the < 35 years old group, and the difference is statistically significant. As reported in other studies, the common CNVs are not related to maternal age(17), and our findings suggest that the positive rate of CNVs with the different maternal age groups is trending but not statistically significant.

The incidence of birth defects in China is about $5.6 \%(18)$. Chromosomal aberrations account for more than $80 \%$ of the genetic causes, including abnormal number of chromosomes, and pathogenic copy number variations (pCNVs, which account for $50 \%)(19)$. So far, more than 300 types of pCNVs have been found to cause chromosome microdeletion/microduplication syndrome, and the comprehensive incidence rate is nearly $1 / 600(20)$. Therefore, effective prenatal screening and subsequent timely prenatal diagnosis for chromosomal aberrations is critical for reducing the birth defects of live births. Expanded NIPT performance in some CNVs has been thoroughly described. A prospective study which involved a large group of pregnant women showed that expanded NIPT exhibited high performance for the 22q11.2 microdeletion, and moderate-to-low performance for detection of other, genome-wide, segmental imbalances associated with other MMS and some CNVs(9). In this study, we found 29 (36.25\%) true-positive cases of chromosomal microdeletions or microduplications that were validated by CNV-sEq. Among the 29 true-positive cases, 13 cases occurred because of genetic mutations. We also found 51 (63.75\%) false-positive cases. Among the 51 false-positive cases, 9(17.65\%) cases occurred because of abnormalities in the maternal peripheral blood, consistent with other literature that showed MCNV can potentially contribute to a small but significant number of false-positive fetal trisomies detected by NIPT(21). NIPT uses cell-free fetal DNA (cffDNA) extracted from maternal plasma, which is a mixture of maternal DNA and a low percentage of fetal DNA. Therefore, chromosomal aneuploidy and CNVs abnormalities of pregnancy have a great influence on NIPT results, making the reliable and accurate detection of aneuploidies or MMS challenging(22). A study reported that altered maternal $X$ chromosome karyotype and maternal X CNVs contribute to discordant NIPT SCAs results(23). In this study, we found 31 (34.06\%) truepositive cases for SCAs that were validated by karyotype and CNV-sEq. We also found eight cases that were detected as normal results in the fetal amniotic fluid, but the FISH test showed SCAs in maternal peripheral blood, which account for $13.33 \%$ of the false positive SCAs cases. From this data, we can conclude that the pregnancy SCAs and CNVs have a great influence on the accuracy of NIPT results. Apart from pregnancy SCAs or CNVs, low fetal DNA fraction and confined placental mosaicism (CPM)(24) can confound any NIPT results.

Many studies have shown that, compared with traditional screening technologies, expanded NIPT has better sensitivity and accuracy for detecting Chromosome aneuploidy $(9,17,25,26)$, and it is feasibility for detecting fetal CNVs. As the depth of sequencing increases and calculation methods change, monogenic diseases, such as congenital adrenal hyperplasia, Duchenne muscular dystrophy (DMD) and others may also be identified via expanded NIPT(27-29). However, it remains some defects such as unable to detect chromosome structural variations, unable to avoid false positives and false negatives, unable to remove the influence of maternal abnormalities until now. Our findings suggest that maternal SCAs and CNVs contribute to a small but significant number of false-positive fetal trisomies and CNVs detected by NIPT. Therefore, to avoid false-positives caused by maternal SCAs or CNVs and avoid unnecessary invasive procedures, we 
recommend that when NIPT indicates a high risk of SCAs and CNVs, pregnancy DNA sequencing should be performed. There is also a need to develop a new analysis or calculation method to remove the potential pregnancy influence on expanded NIPT results.

\section{Conclusion}

Our study shows that expanded NIPT has high sensitivity and specificity in detecting diseases of chromosomal aneuploidy. It also shows good performance in detecting CNVs, but maternal SCAs and CNVs may impact expanded NIPT results, and contribute to a small but significant number of false-positives, thus possibly hindering the use of the expanded NIPT to accurately detect fetal SCAs or CNVs. Therefore, in order to improve the accuracy of detection, there remains a need to remove the potential maternal influence on expanded NIPT results and need relevant clinical validation studies.

\section{Methods}

\subsection{Patients}

This study was designed as a retrospective study, and the inclusion criteria were as follows: (1) single pregnancy, and (2) natural conception. The exclusion criteria were as follows: (1) multiple pregnancy, (2) conception through IVF (in vitro fertilization), (3) received immunotherapy within 4 weeks of NIPT, and (5) first NIPT test failed. According to the above criteria, a total of 16128 pregnant women were recruited from February 2017 to December 2020. Venous blood samples were collected from the Gansu Province Maternal and Child Health Care Hospital in Lanzhou, China. All the participants purchased Taikang insurance under a specific expanded NIPT insurance scheme covering the standard and expanded test range. Informed written consent was obtained from all participants who agreed to receive expanded NIPT. Pregnancies were divided into the following groups: fetal structural abnormalities by ultrasound (including NT $\geq 3 \mathrm{~mm}$ ), high risk of serological screening (T21 > 1/270, T18 > 1/350), advanced maternal age ( $\geq 35$ years), critical risk of serological screening (T21 $1 / 270$ to1/1000, T18 1/350 to1/1000), No serology screening, and no clinical indications (low risk of serological screening, no abnormalities on ultrasound and no advanced maternal age). The study was approved by the hospital ethics committee, and all pregnancy signed an informed consent form.

\subsection{Library construction and DNA sequencing}

We collected 8 to $10 \mathrm{~mL}$ of whole blood samples in special tubes (Streck, USA). Plasma separation was performed at $4^{\circ} \mathrm{C}$ within $72 \mathrm{~h}$ of blood sample collection. Afterwards, cell-free DNA extraction and purification, library construction, quality control, quantification, addition of sequence tags, and pooling were performed according to the fetal chromosome aneuploidies (T21/T18/T13) test kit (Berry Genomics, China). Finally, the samples were sequenced on the NextSeq CN500 platform (Illumina, USA). Sequencing reads were mapped to the human reference genome (GRCh37/hg19). Sequencing and analysis were performed as previously described(9).

\subsection{Prenatal diagnosis}

Each participant received counselling after expanded NIPT screening. Positive expanded NIPT individuals were recommended to receive invasive prenatal diagnosis. Invasive prenatal diagnosis and follow-up results were used as the gold standard to calculate the true positive case. Whole chromosomal aneuploidies were confirmed by karyotyping and CNVs were confirmed by CNV-SEq. The pathogenicity of CNVs were evaluated following the ACMG guidelines.

\subsection{Peripheral blood test}

Study participants with sex chromosomal abnormalities (SCAs) detected by expanded NIPT were also recommended to receive a peripheral blood FISH test. Participants with CNVs detected by expanded NIPT were also recommended to receive Parents' peripheral blood CNVSEq. The pathogenicity of the CNVs were evaluated following the ACMG guidelines.

\section{Abbreviations}

NIPT

Noninvasive prenatal testing

CNVs

Copy number variants

TPR

The true positive rate

FPR 
The false positive rate

SCAs

Sex chromosome abnormalities

cffDNA

The cell-free fetal DNA

MMS

Microdeletion/microduplication syndromes

PPV

Positive predictive value

MCNV

Maternal copy number variants

pCNVs

Pathogenic copy number variations

CPM

Confined placental mosaicism

DMD

Duchenne muscular dystrophy

IVF

In vitro fertilization

\section{Declarations}

\section{Ethics approval and consent to participate:}

This study was approved by the Gansu Province Maternal and Child Health Care Hospital ethics committee

\section{Consent for publication:}

Not applicable

\section{Availability of data and materials:}

The datasets used and analyzed during the current study are available from the corresponding author on reasonable request.

\section{Competing interests:}

The authors declare that they have no competing interests.

\section{Funding:}

This study was supported by Lanzhou Science and Technology Bureau Project (2017-4-50).

\section{Authors' contributions:}

Jing He conceived the idea and as a major contributor in writing the manuscript, as well as analyzed the data; Sheng-ju Hao, who funded the study, is responsible for the quality control; Xuan Feng and Xing Wang oversight and leadership responsibility for the research activity planning and execution, also responsible for data collection; Qing-hua Zhang and Lei Zheng were in charge of the patient's informed consent and genetic counseling for pregnant; Peng-wu Lin responsible for follow-up of pregnant. All authors read and approved the final manuscript.

\section{Acknowledgments}

We thank all of the project participants for their contributions.

\section{References}

1. Lo Y, Corbetta N, Chamberlain PF, Rai V, Wainscoat JS. Presence of fetal DNA in maternal plasma and serum. Lancet. 1997;350(9076):485-7. 
2. Fan HC, Blumenfeld YJ, Chitkara U, Hudgins L, Quake SR. Noninvasive diagnosis of fetal aneuploidy by shotgun sequencing DNA from maternal blood. Proc Natl Acad Sci USA. 2008;105(42):16266-71.

3. Srebniak MI, Knapen M, Govaerts L, Polak M, Opstal DV. Social and medical need for whole genome high resolution NIPT. 8: Molecular Genetics \& Genomic Medicine; 2020.

4. Neofytou MC, Kyriakos T, Elena K, Charalambos L, Marios I, Achilleas A, et al. Targeted capture enrichment assay for non-invasive prenatal testing of large and small size sub-chromosomal deletions and duplications. Plos One. 2017;12(2):e0171319.

5. Quezada MS, Gil MM, Francisco C, Oròsz G, Nicolaides KH. Screening for trisomies 21, 18 and 13 by cell-free DNA analysis of maternal blood at 10-11 weeks' gestation and the combined test at 11-13 weeks. Ultrasound Obstet Gynecol. 2015;45(1):36-41.

6. Yaron Y, Jani J, Schmid M, Oepkes D. Current Status of Testing for Microdeletion Syndromes and Rare Autosomal Trisomies Using CellFree DNA Technology. Obstetrics Gynecology. 2015;126(5):1095-9.

7. Srebniak MI, Joosten M, Knapen M, Arends LR, Opstal DV. Frequency of submicroscopic chromosome aberrations in pregnancies without increased risk for structural chromosome aberrations: a systematic review of literature and meta-analysis. Ultrasound in Obstetrics and Gynecology. 2017;51(4).

8. Watson CT, Tomas MB, Sharp AJ, Me Ff Ord HC. The Genetics of Microdeletion and Microduplication Syndromes: An Update. Annual Review of Genomics Human Genetics. 2014;15(1):215.

9. Liang D, Cram DS, Tan H, Linpeng S, Liu Y, Sun H, et al. Clinical utility of noninvasive prenatal screening for expanded chromosome disease syndromes. Genetics in Medicine. 2019.

10. Gregg AR, Skotko BG, Benkendorf JL, Monaghan KG, Bajaj K, Best RG, et al. Noninvasive prenatal screening for fetal aneuploidy, 2016 update: a position statement of the American College of Medical Genetics and Genomics. Genetics in Medicine; 2016.

11. Mackie F, Hemming K, Allen S, Morris R, Kilby M. The accuracy of cell-free fetal DNA-based non-invasive prenatal testing in singleton pregnancies: a systematic review and bivariate meta-analysis. Bjog. 2017.

12. Obstetricians ACo, Bulletins-Obstetrics GCoP, Genetics CO, Medicine MF. Practice Bulletin No. 162: Prenatal Diagnostic Testing for Genetic Disorders. Obstetrics Gynecology. 2016;127(5):e108.

13. Khalifeh A, Weiner S, Mackeen D, Gerson A, Alan D. 562: Trends in invasive prenatal diagnosis from 2002-2011: a population-based evaluation. American Journal of Obstetrics Gynecology. 2013;208(1):241-S.

14. Meij K, Sistermans EA, Macville M, Stevens S, Weiss MM. TRIDENT-2: National Implementation of Genome-Wide Non-Invasive Prenatal Testing as a First-Tier Screening Test in the Netherlands. The American Journal of Human Genetics. 2019;105(6).

15. Chan T, Tao, Deng, Xiuhuang, Zhu, et al. Evidence of compliance with and effectiveness of guidelines for noninvasive prenatal testing in China: a retrospective study of 189,809 cases. Science China(Life Sciences). 2020;63(03):5-14.

16. Franasiak JM, Forman EJ, Hong KH, Werner M, Upham KM, Treff NR, et al. The nature of aneuploidy with increasing age of the female partner: a review of 15,169 consecutive trophectoderm biopsies evaluated with comprehensive chromosomal screening. Fertility Sterility. 2014;101(3):656 - 63.e1.

17. Chen Y, Yu Q, Mao X, Lei W, He M, Lu W. Noninvasive prenatal testing for chromosome aneuploidies and subchromosomal microdeletions/microduplications in a cohort of 42,910 single pregnancies with different clinical features. Human Genomics. $2019 ; 13$.

18. Chen Y, Xu Q, Schweitzer SO. Use of genetic services for the prevention and comtol of birth defects in China. Journal of Anhui Vocational College of Police Officers. 2005;8(5):1-5.

19. Evans MI, Wapner RJ, Berkowitz RL. Noninvasive prenatal screening or advanced diagnostic testing: caveat emptor. American Journal of Obstetrics \& Gynecology. 2016:298-305.

20. Nevado J, Mergener R, Palomares-Bralo M, Souza KR, Vallespín E, Mena R, et al. New microdeletion and microduplication syndromes: a comprehensive review. Genetics Molecular Biology. 2014;37(1 Suppl):210-9.

21. Xiya Zhou LS, Xu Y, Song Y, Qi Q, Zhang J, Zhu H, Sun H, Tian F, Xu M, Cram DS. Juntao Liu. Contribution of maternal copy number variations to false-positive fetal trisomies detected by noninvasive prenatal testing. Prenatal Diagnosis. 2017;37(4).

22. Ad Vani HV, Barrett AN, Evans MI, Choolani M. Challenges in non-invasive prenatal screening for sub-chromosomal copy number variations using cell-free DNA. Prenat Diagn. 2017.

23. Shaowei Wang SH, Linlin Ma L, Liang J, Zhang J, Zhang DS. Cram Maternal X chromosome copy number variations are associated with discordant fetal sex chromosome aneuploidies detected by noninvasive prenatal testing. Clin Chim Acta. 2015;444:113-6.

24. Grati FR, Malvestiti F, Ferreira J, Bajaj K, Simoni G. Fetoplacental mosaicism: potential implications for false-positive and false-negative noninvasive prenatal screening results. Genetics in Medicine Official Journal of the American College of Medical Genetics. 2014;16(8).

25. Hu H, Wang L, Wu J, Zhou P, Fu J, Sun J, et al. Noninvasive prenatal testing for chromosome aneuploidies and subchromosomal microdeletions/microduplications in a cohort of 8141 single pregnancies. Human Genomics. 2019;13(1).

Page 11/12 
26. Yu D, Zhang K, Han M, Pan W, Chen Y, Wang Y, et al. Noninvasive prenatal testing for fetal subchromosomal copy number variations and chromosomal aneuploidy by low-pass whole-genome sequencing. Molecular Genetics \& Genomic Medicine; 2019.

27. Luo Y, Jia B, Yan K, Liu S, Song X, Chen M, et al. Pilot study of a novel multi-functional noninvasive prenatal test on fetus aneuploidy, copy number variation, and single-gene disorder screening. Molecular Genetics \& Genomic Medicine; 2019.

28. New MI, Tong YK, Tony Y, Jiang P, Christian P, Chan K, et al. Noninvasive prenatal diagnosis of congenital adrenal hyperplasia using cellfree fetal DNA in maternal plasma. J Clin Endocrinol Metab. 2014(6):E1022.

29. Xu Y, Li X, Ge HJ, Xiao B, Zhang YY, Ying XM, et al. Haplotype-based approach for noninvasive prenatal tests of Duchenne muscular dystrophy using cell-free fetal DNA in maternal plasma. Genetics in Medicine Official Journal of the American College of Medical Genetics. 2015.

Figures

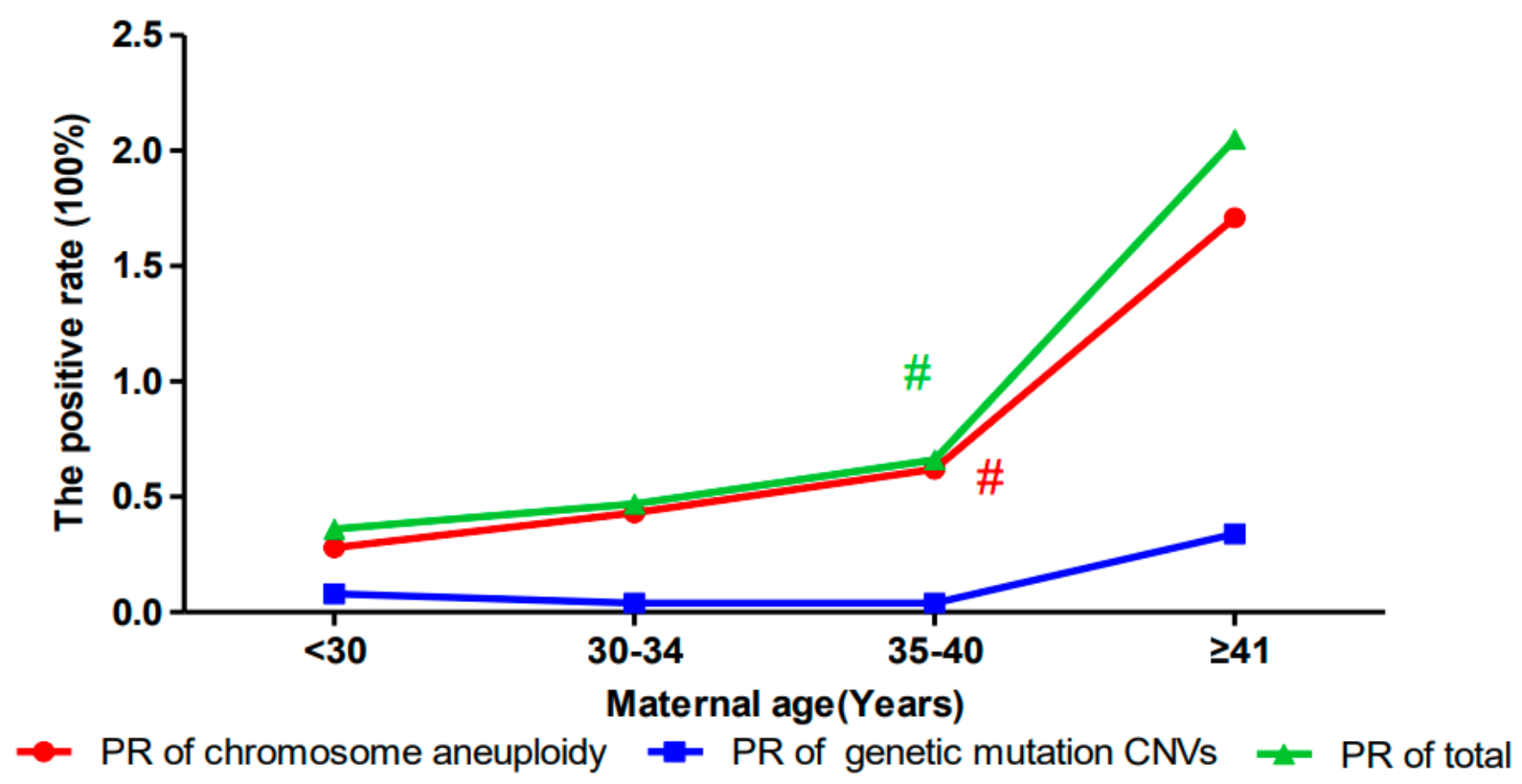

Figure 1

The positive rate of expanded NIPT increased with maternal age in the four age groups. \# vs (<30 group add 30-34 group). Chi-square test was used to test the significance of the differences between the different groups. For aneuploidies, the positive rate of advance maternal age (35-40 group add $\geq 41$ group) group is higher than $<35$ group ( $<30$ group add 30-34 group), and the high was statistically significant $(\chi 2=8.651, p=0.003<0.05)$. For $C N V$, the difference between the advance maternal age (35-40 group add $\geq 41$ group) add $<35$ group $(<30$ group add 30-34 group) was not statistically significant $(\chi 2=0.000, p=1.000>0.05)$. The total positive rate increased with maternal age, the positive rate of advance maternal age ( $35-40$ group add $\geq 41$ group) group is higher than $<35$ group ( $<30$ group add $30-34$ group), and the high was statistically significant $(\chi 2=4.409, p=0.036<0.05)$. 